\title{
Water resistant nanopapers prepared by lactic acid modified cellulose
}

\section{nanofibers}

9 Abstract

10 The current work reports a novel, completely water based approach to prepare the water resistant
Jatin Sethi ${ }^{1}$, Muhammad Farooq ${ }^{1}$, Sunanda Sain ${ }^{1}$, Mohini Sain ${ }^{2,3}$, Juho Antti Sirviö $^{1 *}$, Mirja Illikainen ${ }^{1}$ and Kristiina Oksman ${ }^{1,2,3}$ ${ }^{1}$ Fibre and Particle Engineering Research Unit, University of Oulu, Oulu, Finland. ${ }^{2}$ Division of Materials Science, Luleå University of Technology, Luleå, Sweden. ${ }^{3}$ Centre for Biocomposites and Biomaterials Processing, University of Toronto, Toronto, Canada. ${ }^{*}$ Corresponding author: Juho Antti Sirviö, Email: juho.sirvio@oulu.fi modified cellulose nanopapers. Lactic acid in aqueous medium was attached on cellulose nanofibers

surface with the aid of ultra-sonication and later oligomerized (polymerized) by compression molding

under high temperature and pressure, to obtain the modified nanopapers with enhanced mechanical

4 properties. The modified nanopapers showed an increase of $32 \%$ in the elastic modulus and $30 \%$ in the

5 yield strength over reference nanopapers. Additionally, the modified nanopaper was hydrophobic in

nature and had superior storage modulus under moist conditions. The storage modulus of wet modified

nanopaper was three times $(2.4 \mathrm{GPa})$ compared to the reference nanopapers $(0.8 \mathrm{GPa})$ after 1 hour

immersion in water. Finally, the thermal stability of the modified nanopaper was also higher than

reference nanopaper. The material reported is $100 \%$ bio-based.

0 Keywords: Cellulose Nanofibers, Lactic acid, Water resistant Nanopaper, Mechanical properties

\section{Introduction}

The growing human population and increasing consumption has resulted in excessive use of our non-

3 renewable natural resources. This demands the development of new and more sustainable materials 


\section{Page 2 of 21}

24 from renewable resources. Cellulose, the most abundant renewable biomaterial in the world, has been

25 widely studied as a raw material for new biomaterials, especially as a form of nano-sized cellulose

26 crystals (CNCs) and fibers (CNFs). Nanocellulose has astonishing mechanical properties: an elastic

27 modulus of around $150 \mathrm{GPa}$ (Lee et al. 2014) and for that reason it has widely been studied as

28 reinforcements for polymers (Lee et al. 2014), or as a sheet-like material termed as nanopaper (Sehaqui

29 et al. 2012).

30 Nanopapers are prepared by removing water from nanocellulose suspensions, usually by vacuum

31 filtration or evaporation. The morphology of the nanopapers indicate an intricate network of cellulose

32 nanofibers, which high amount of interfibrillar bonding due to presence of hydroxyl groups on

33 cellulose molecules. This bonding results in excellent mechanical properties; it has been demonstrated

34 that nanopapers can have modulus of 9.4-14 GPa and strength of 103-449 MPa (Lee et al.

35 2014)(Sehaqui et al. 2012). With such excellent properties and renewable nature, nanopapers have

36 raised themselves as potential replacement for non-renewable materials applications such as food

37 packaging and electronic displays (Sehaqui et al. 2014).

38 Despite high strength of the nanopapers, there exists a fundamental weakness: they lose their strength

39 in damp conditions. Sehaqui et al. portrays this problem in their work, they found that in wet state

40 tensile modulus of nanopapers was only $5 \%$ of the value in dry state (Sehaqui et al. 2014). Even in high

41 humidity of $95 \%$, the storage modulus was $25 \%$ of storage modulus in dry conditions. The reason

42 behind this was explained by the fact that in presence of water molecules, the interfibrillar bonds

43 between fibers are heavily weakened (Sehaqui et al. 2014). Due to absence of interfibrilar bond the

44 fibers easily slides under external stress resulting diminished strength to nanopaper. Additionally, water

45 molecules plasticizes the amorphous regions of cellulose (Benítez et al. 2013). This inability to combat

46 moisture negates the positive advantages of high strength nanopaper. Hence, it is of interest to prepare 


\section{Page 3 of 21}

47 the water resistant nanopapers. In fact, it has been proposed that commercial viability of nanopapers

48 can only be ensured if they have enhanced mechanical properties in the presence of water (absorbed

49 moisture or liquid) (Benítez et al. 2013).

50 The hydrophilicity of cellulose surface and its ability to absorb water can be altered by chemically

51 modifying the surface of nanocellulose. The functionalization of cellulose has been reported by using

52 two mechanisms: covalent grafting of chemical entities and physical adsorption on the cellulose surface

53 (Tingaut et al. 2012). Covalent grafting is a common approach to improve hydrophobicity of cellulose

54 nanofibrils. Sehaqui et al. prepared hydrophobic nanopapers by modifying cellulose by mild

55 esterification by alkyl chains, which showed decreased moisture intake (Sehaqui et al. 2014).

56 Additionally, the wet strength was improved as much as 24 times when compared to reference.

57 However, the esterification is usually done in organic solvent or monomer medium (Habibi 2014;

58 Sehaqui et al. 2014), which are hazardous and costly. Therefore, use of water as medium is of great

59 practical value.

60 This work uses esterification of CNF surface with lactic acid in water medium. Although, the

61 esterification of CNFs is not a vastly studied topic, few researchers have published relevant results in

62 solvent medium (Lönnberg et al. 2006)(Peltzer et al. 2014) (Teramoto et al. 2002). Esterification is a

63 dehydration reaction which is often is not feasible in water medium, as the reaction product itself is a

64 water molecule. The product water is in equilibrium with medium water and reaction is not preferred

65 due to law of mass action (Kobayashi et al. 1997). However, esterification in water medium has been

66 done with the help of catalysts. (Kobayashi et al. 1997) used lipase based enzyme for

67 polycondensation, and (Tanaka and Kurihashi 2003) used dodecylbenzenesulfonic acid as catalyst and 68 surfactant. 


\section{Page 4 of 21}

69 This work attempts a novel approach to prepare esterified CNF nanopapers, which includes the

70 ultrasonication of CNFs in presence of lactic acid in water medium. Chemical reactions with the aid of

71 acoustic energy relates to the field of sonochemistry. During sonication, cavities (vacuum pockets) are

72 formed which have short life time. The cavities implode at an extremely high speed to form hotspots

73 which have high temperatures and pressures, (5000 K and pressure of 1000 atmospheres). The process

74 is known as cavitation. Such extreme conditions can produce chemical reactions that may otherwise not

75 happen (Suslick 2000). After ultrasonication, modified nanopapers were made by removal of water

76 under vacuum filtration and further processing at high temperature $\left(150{ }^{\circ} \mathrm{C}\right)$ and pressure $(10 \mathrm{MPa})$.

77 The samples were characterized by tensile testing, swelling studies, dynamic mechanical analysis

78 (DMA) under varying moisture and thermogravimetric analysis (TGA).

\section{Experimental}

\section{$80 \quad 2.1 \quad$ Materials}

81 L-(+)-Lactic acid (80\%) was purchased from Sigma-Aldrich. Stannous chloride (Merck Millipore) was 82 purchased from VWR. Bleached soft wood sulfite fibers were kindly supplied by Stora Enso (Oulu,

83 Finland). The pulp (1.6 wt. \%) was grinded in Masuko grinder. The pulp was repeated fed in contact 84 mode from 0-point, and distance was gradually decreased from - 20 (3 passes), - 40 (4 passes), -60 (5 85 passes) and -90 (7 passes). The chemical composition of the reference pulp was $95.0 \mathrm{wt} \%$ cellulose, 4.2 $86 \mathrm{wt} \%$ hemicellulose, $0.3 \mathrm{wt} \%$ lignin and $0.5 \mathrm{wt} \%$ inorganics.

$87 \quad 2.2 \quad$ Modification of nanofibers and processing of nanopapers

88 After the fibrillation, cellulose nanofibers were diluted to the concentration of $0.4 \mathrm{wt} \%$ and LA was 89 added according to formulation given in Table 1. It is worth mentioning that tests were conducted with 90 different level of LA, it was found that there is no significant effect on the properties of modified

91 nanopapers (especially modulus), perhaps due to the amount of LA attached to the surface of 


\section{Page 5 of 21}

92 nanocellulose was not different; therefore for the purpose of simplicity, only a relevant formulation is

93 reported in this study. Stannous chloride was added in trace amounts as catalyst for esterification (Yoo

94 et al. 2006). The suspensions were stirred at $9000 \mathrm{rpm}$ in Ultra-turrax homogenizer for 5 minutes, and

95 further sonicated with the help of probe type sonicator (Heilscher UP 400s). The sonication was

96 stopped when the sonication energy reached $1100 \mathrm{~J} / \mathrm{ml}$. After the sonification the suspensions were

97 kept in the oven at $100{ }^{\circ} \mathrm{C}$ for 36 hours. The schematics are presented in Figure 1 . These suspensions,

98 henceforth mentioned as LA modified CNFs, were used to make nanopapers.

Table 1 Formulations of CNF suspensions with lactic acid before sonication

Sample name

\begin{tabular}{lcc}
\hline & \multicolumn{2}{c}{ Sample name } \\
\hline Materials & Reference nanopaper & Modified nanopaper \\
\hline CNF & 0.4 & 0.4 \\
L-Lactic acid & 0 & 7.6 \\
SnCl & & 0.0002 \\
Water & 0 & 92 \\
\hline Total & 99.6 & $100 \mathrm{wt} . \%$ \\
\hline
\end{tabular}

101 Nanopapers (reference and modified) were prepared by diluting LA modified CNFs and reference CNF

102 suspension to $0.2 \mathrm{wt} \%$ by vacuum filtering through a Durapore PVDF membrane filter (Fisher

103 Scientific, Pittsburgh, USA) with a pore size of $0.65 \mu \mathrm{m}$ (Figure 1). The suspensions were degassed

104 under the vacuum of $70 \mathrm{kPa}$ for half an hour before the filtering. After the vacuum filtration, the wet

105 cellulosic sheet was carefully peeled from the membrane and kept between two steel mesh cloths (mesh

106 size $70 \mu \mathrm{m}$ ), which were further stacked in paper carrier board. The CNF sheet-steel mesh-paper board

107 was kept under compression molding plates at temperature of $100{ }^{\circ} \mathrm{C}$ at the pressure of $10 \mathrm{MPa}$ for 30 


\section{Page 6 of 21}

108 minutes to remove water. The LA-grafted nanopapers were further compression molded at $150^{\circ} \mathrm{C}$ for 5

109 minutes, to increase the yield of esterification. In order to take account the effect of the sonication, two

110 type of references were prepared, one sonicated suspension and one without sonication. All the

111 nanopapers were stored in ambient conditions for 72 hours before testing. The grammage (weight in

112 grams per square meters) of nanopapers was between 27-30 gsm.

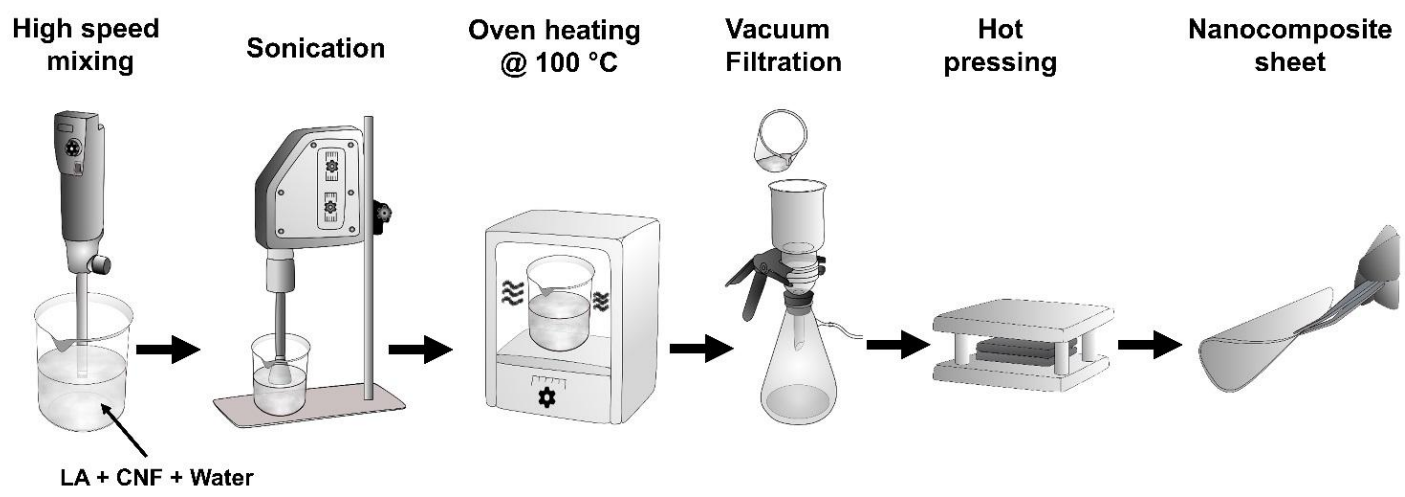

Figure 1 Schematic diagram of process used for preparation of nanopapers

$115 \quad 2.3$ Characterization

116 Diffuse reflectance infrared Fourier transform (DRIFT) spectroscopy was used to confirm the

117 esterification of CNFs. The filtered wet cake from nanopaper preparation was rolled into a spherical

118 shape and kept in oven at $90-95{ }^{\circ} \mathrm{C}$ overnight to remove water. The modified CNFs were further kept at

$119150{ }^{\circ} \mathrm{C}$ for half an hour. It was done as because the restriction from the equipment that FTIR data from

120 thin nanopapers was not feasible. The hardened reference and modified CNFs were grinded into

121 powder, and the spectra were collected from dried samples with Bruker Vertex $80 \mathrm{~V}$ spectrometer

122 (USA), in the 400-4000 $\mathrm{cm}^{-1}$ range, and 40 scans were taken at a resolution of $4 \mathrm{~cm}^{-1}$ for each

123 sample.

124 Wide angle X-ray diffraction (WAXRD) was used to determine the crystalline structure of the

125 reference and the modified nanopaper. Rigaku SmartLab 9kW rotating anode diffractometer (Japan) 


\section{Page 7 of 21}

126 using a Co K $\alpha$ radiation $(40 \mathrm{kV}, 135 \mathrm{~mA} ; \lambda=1.79030 \AA)$ was used for measurements. Bragg’s angle

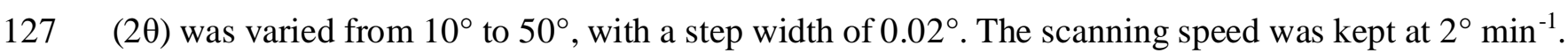

128 The degree of crystallinity (CrI) was calculated from the peak intensity of the main crystalline plane

129 (200) diffraction ( $\mathrm{I}_{200}$ ) which was at $26.2^{\circ}$ and from peak intensity at $22^{\circ} \mathrm{C}$, which is associated to

130 amorphous fraction of cellulose $\left(\mathrm{I}_{\mathrm{am}}\right)$ (French 2014), according to the Equation 1:

$$
\mathrm{CrI}=\left(\frac{\mathrm{I}_{200}-\mathrm{I}_{\mathrm{am}}}{\mathrm{I}_{200}}\right)
$$

Equation 1

131 We would like to mention that due to the Co K $\alpha$ radiation source, the peak of cellulose have different

132 diffraction angles compared to the peaks obtained for $\mathrm{Cu} \mathrm{K} \alpha$ radiation source.

133 The average size of crystallite (L) was calculated from the Scherrer equation (Ahtee et al. 1983) :

$$
L=\frac{K \times \lambda}{\beta \times \cos \theta}
$$

136 where $\mathrm{K}$ is a constant value $0.74, \lambda$ is the $\mathrm{X}$-ray wavelength $(0.17903 \mathrm{~nm}), \beta$ is the half-height width of 137 the diffraction band (200); and $\theta$ is the Bragg angle corresponding to the (200) plane.

138 Mechanical testing was done to evaluate tensile properties of the CNF networks using Instron 5544 139 universal material testing machine (Norwood, USA). Strips with a length of $50 \mathrm{~mm}$ and a width of 5 $140 \mathrm{~mm}$ were conditioned at $23^{\circ} \mathrm{C}$ and relative humidity $(\mathrm{RH})$ of $50 \%$ for $72 \mathrm{~h}$ prior to the testing. A load 141 cell of $100 \mathrm{~N}$ was used, the crosshead speed was $2 \mathrm{~mm} / \mathrm{min}$ and the gauge length of $30 \mathrm{~mm}$. The tests 142 were conducted in special chamber maintained at a RH $50 \%$ and in the temperature of $23{ }^{\circ} \mathrm{C}$. The 143 elastic modulus (E) was determined from slope in linear region and yield strength $\sigma_{0.2}$ was determined 


\section{Page 8 of 21}

144 by intersection of $0.2 \%$ offset line and stress strain curve. The results are reported as average of 145 minimum 5 samples.

146 Zeiss Ultra Plus (Oberkochen, Germany) field emission scanning electron microscopy (FE-SEM) was 147 used for analysis of fiber-polymer network morphology of the samples. The acceleration voltage of 3 $148 \mathrm{kV}$ was used. The samples were coated with platinum to avoid charging. Inlens detector was used to 149 collect the signals for imaging.

150 Dynamic mechanical analysis (DMA) under variable humidity was conducted in order to determine the 151 effect of humidity on storage modulus of reference and modified nanopaper using DMA Q800, TA 152 Instruments (New Castle, USA) (equipped with RH accessory) along with strain mode with amplitude 153 of $10 \mu \mathrm{m}$ and frequency of $1 \mathrm{~Hz}$. The samples were equilibrated at $30{ }^{\circ} \mathrm{C}$ and $0 \%$ relative humidity for 1542 hours to remove the absorbed moisture. Subsequently, relative humidity was raised from $0 \%$ to $95 \%$ 155 at the rate of $1 \% / \mathrm{min}$ and the storage modulus was recorded. The mechanical properties in wet 156 condition were studied using same equipment and the isothermal tests were conducted in tension mode 157 at an amplitude of $20 \mu \mathrm{m}$ and using a frequency of $1 \mathrm{~Hz}$.

158 Thermogravimetric analysis (TGA) of the nanopapers (reference and modified) was conducted in order 159 to determine the thermal stability of samples using TA-TGA Q500 (New Castle, USA). Sample weight 160 around $10 \mathrm{mg}$ was kept on a hanging platinum pan and heated till the temperature of $900{ }^{\circ} \mathrm{C}$ under the 161 nitrogen atmosphere, with the heating rate of $10^{\circ} \mathrm{C} / \mathrm{min}$. The moisture content of samples was 162 determined by weight loss between $0-200{ }^{\circ} \mathrm{C}$. 


\section{Page 9 of 21}

1633 Results and discussion

$164 \quad 3.1 \quad$ Modification of CNFs

165 The esterification reaction between hydroxyl groups of CNF and carboxylic groups of LA was

166 confirmed by FTIR is shown in Figure 2. A peak around $1750 \mathrm{~cm}^{-1}$ (indicated by dotted line in Figure

167 2) can be seen in modified nanopaper sample, which indicates the presence of ester bond (Tjeerdsma 168 and Militz 2005). A small peak can already be observed in FTIR spectra after sonication treatment of 169 CNFs with lactic acid (see Figure 1 supplementary information). High temperature and pressure on 170 nanopapers (after water removal) was used to shift the reaction towards higher conversion.

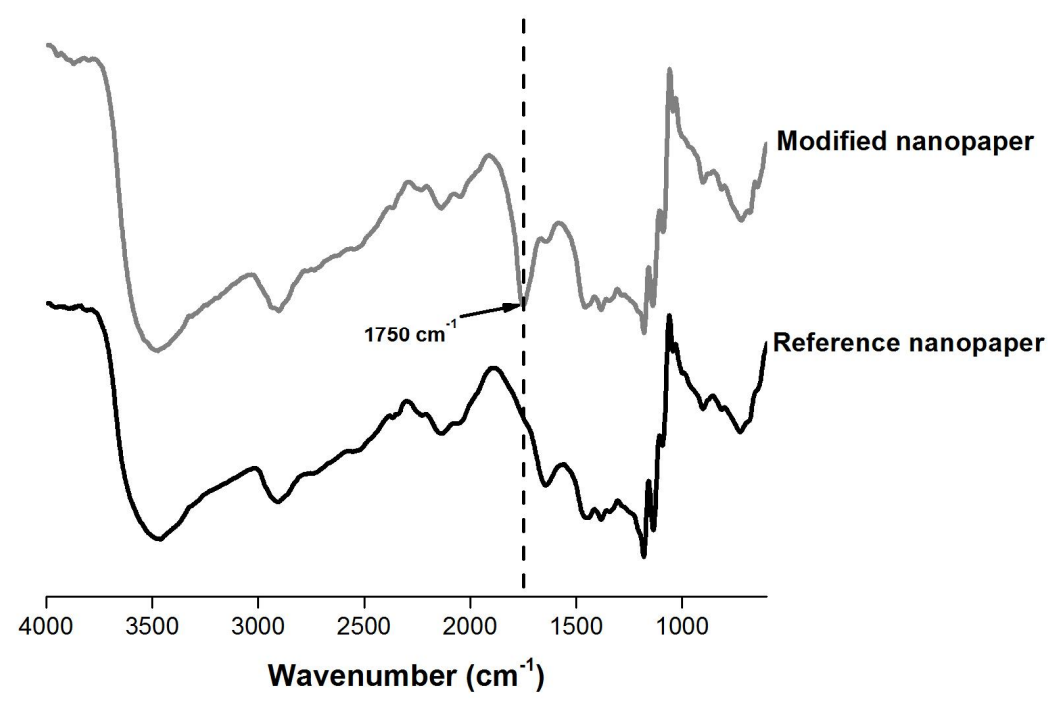

171

172 Figure 2 FTIR spectra of reference and modified nanopapers. The esterification peak is marked with 173 dotted line around $1750 \mathrm{~cm}^{-1}$

$175 \quad 3.2 \quad$ Crystal structure

176 Effect of LA modification on crystal structure of nanopaper was studied using WAXRD and the

177 patterns of reference and modified nanopaper is shown in Figure 3. Both samples exhibited typical 


\section{Page 10 of 21}

178 cellulose I crystalline structure (French 2014). CrI calculated by Segal equation (Equation 1) indicated

179 different amount of crystallinity between samples $(79.5 \%$ and $47.8 \%$ for modified nanopaper and

180 reference, respectively). However, from the Figure 3 it can be seen that relative heights between 1-

$18110 / 110$ doublet and the 200 main peak varies significantly between samples. This might indicate that

182 there is substantial preferred orientation of the samples, caused be sample fabrication. The presence of

183 preferred orientation can have significant effect on the CrI calculation (Park et al. 2010). To verify this,

184 attempt to grind the samples was performed, however, due to the film-like appearance no conventional

185 grinding was successfully and intensively grinding using cryomill severally damaged the crystallinity

186 of the samples. However, based on the Scherrer equation (Equation 2), both samples exhibited similar

187 crystallite size (around $3 \mathrm{~nm}$ ), indicating that no significant amount damage on the cellulose crystals

188 was caused by ultrasonic treatment.

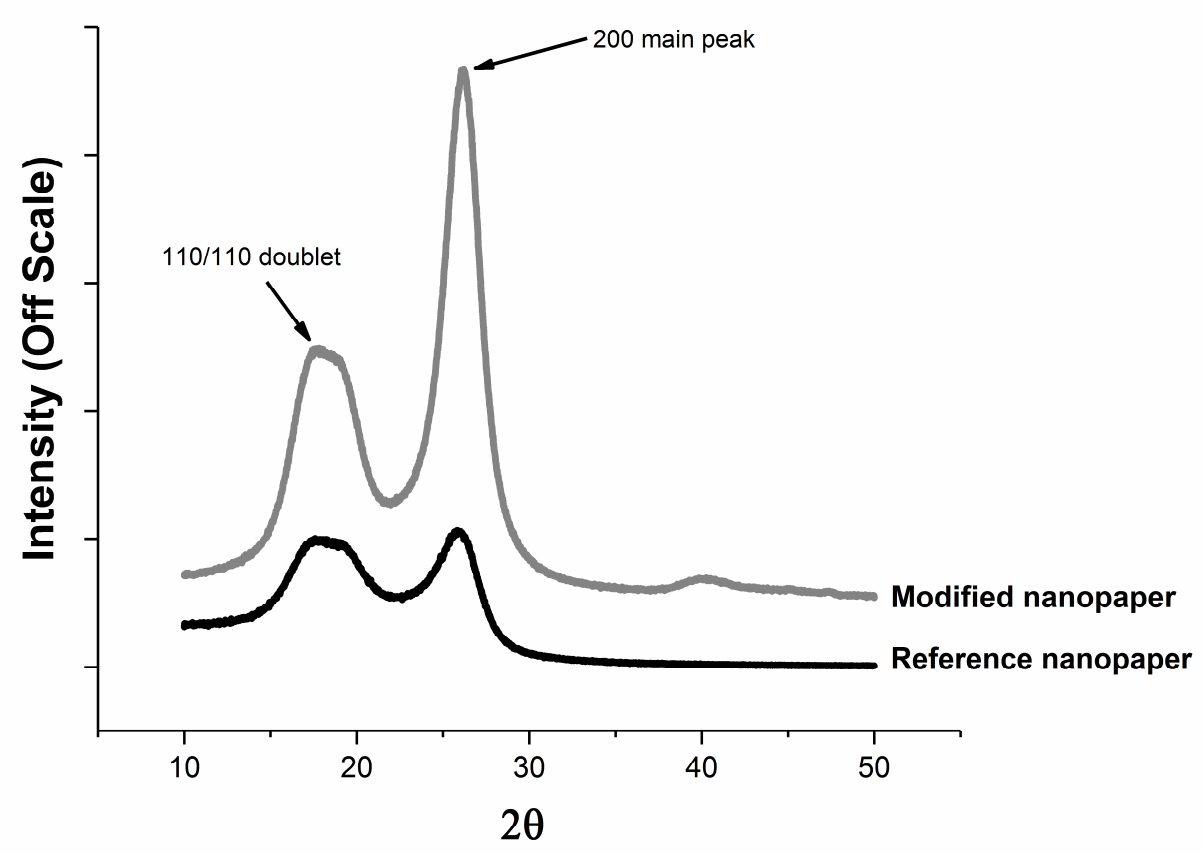




\section{Page 11 of 21}

$191 \quad 3.3 \quad$ Mechanical properties

192 Compared to reference nanopaper, modified nanopaper had higher modulus (Figure 4). The increase is 193 around $32 \%$ (quantitative results are presented in Table 2). It can be speculated that the LA under the 194 high temperature of $150{ }^{\circ} \mathrm{C}$ and pressure of $10 \mathrm{MPa}$ is polymerizing (or oligomerizing), as it has been 195 reported that high temperature and pressure advances the esterification reaction (Ballard et al. 1961).

196 The hydroxyl group from cellulose are also likely to participate in the reaction and forming a strong

197 covalent bond and entire structure can be considered as one rigid hybrid network where hydrogen

198 bonding of CNFs is replaced by covalent bonds, which is restricting movement of CNFs in external

199 load and hence the elastic modulus is increasing. The reaction scheme is provided in Figure 5.

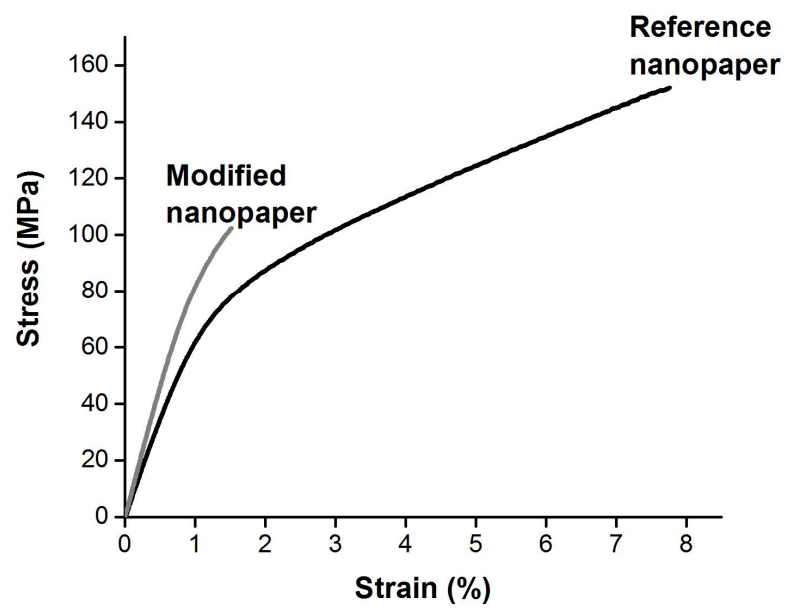




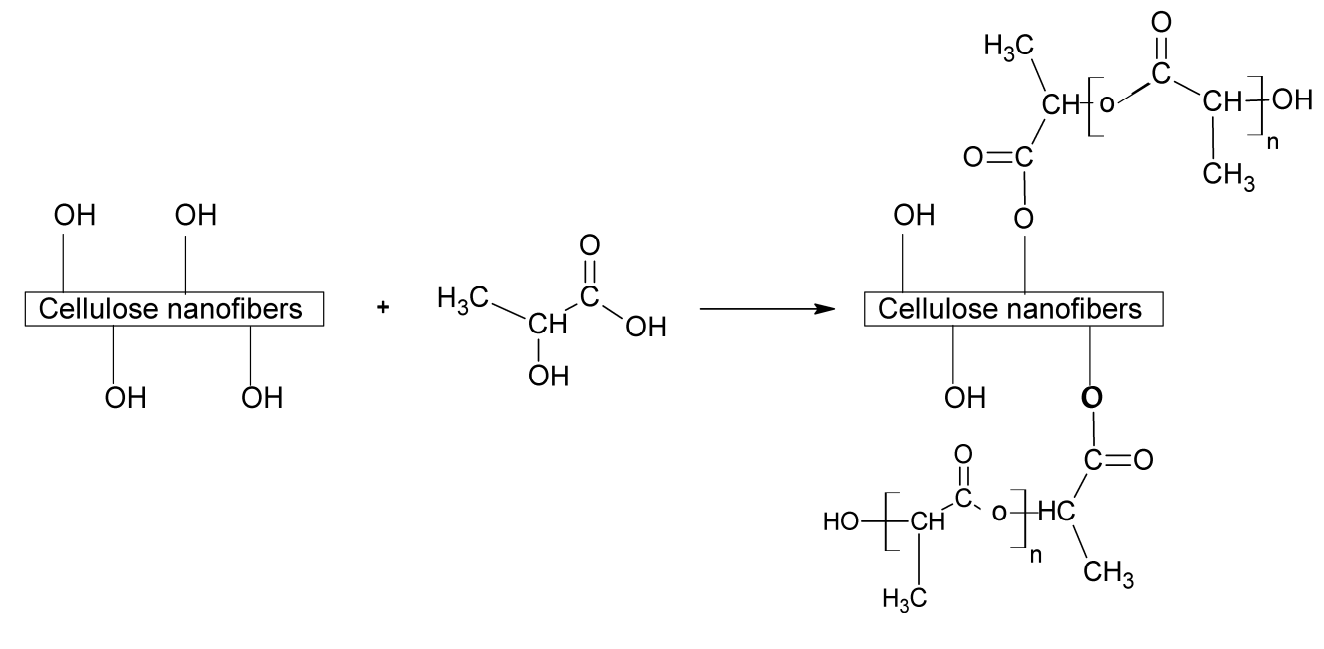

Figure 5 Reaction scheme of CNF with LA under high pressure and temperature.

206 It should also be observed that the yield strength is improved in modified nanopaper by $30 \%$ (Table 2).

207 The strength, determined by interfibrillar sliding (Benítez et al. 2013), implies that the modified

208 nanopapers are more resistant to permanent deformation. This restricting of chains is clearly observed

209 in tensile testing fractured samples in Figure 7, where the reference nanopaper has separated fibrils at

210 fractured cross-section, on the other hand, in modified nanopapers the fibers are heavily bonded to each

211 other. The results implies that modifying the surface of CNFs can be beneficial in making stiffer

212 nanopapers.

213 The effect of sonication on cellulose was also studied. As mentioned before, sonication is an intensive

214 technique and provides energy of 10-100 KJ/mol (Suslick 2000; Tischer et al. 2010), which is of order

215 of hydrogen bonding (Tischer et al. 2010; Przybysz et al. 2016). Wang et al. concluded that sonication

216 can cause structural change in cellulose fibril by causing defibrillation (Wang and Cheng 2009).

217 Therefore, our first step was to analyze the effect of sonication by preparing a nanopaper with same

218 amount of sonication as modified samples. The results is presented in Table 2: the elastic modulus (E)

219 of sonicated nanopaper is approximately $5 \%$ less than non-sonicated one. However, it is worth 


\section{Page 13 of 21}

220 mentioning that the sonicated reference nanopaper has higher density (5\%) so loss in modulus is more

221 marked when density is considered.

222 Finally, the results of this study are particularly outstanding as modified nanopaper reported is $100 \%$

223 bio-based, offer diverse opportunities as applications such as packaging.

Table 2 Quantitative results from stress strain analysis enlisting values (along with standard deviation) elastic modulus, elongation to break, tensile strength, toughness and yield strength of reference and modified nanopapers.

\begin{tabular}{ccccccc}
\hline Materials & $\begin{array}{c}\text { E- } \\
\text { modulus* } \\
(\mathrm{GPa})\end{array}$ & $\begin{array}{c}\text { Elongation } \\
\text { to break* } \\
(\%)\end{array}$ & $\begin{array}{c}\text { Tensile } \\
\text { strength* }^{*} \\
(\mathrm{MPa})\end{array}$ & Toughness* $^{*}$ & $\begin{array}{c}\text { Yield } \\
\text { strength } \\
(\mathrm{MPa})\end{array}$ & $\begin{array}{c}\text { Density } \\
(\mathrm{gm} / \mathrm{cm} 3)\end{array}$ \\
\hline $\begin{array}{c}\text { Reference } \\
(\text { Unsonicated }) \\
\text { Reference }\end{array}$ & $6.8(0.6)^{\mathrm{a}}$ & $8.7(1.1)^{\mathrm{a}}$ & $170(18)^{\mathrm{a}}$ & $985(219)^{\mathrm{a}}$ & $80(5)^{\mathrm{a}}$ & 1.34 \\
$\begin{array}{c}\text { (Sonicated) } \\
\text { Modified }\end{array}$ & $6.4(0.3)^{\mathrm{a}}$ & $11(2.5)^{\mathrm{b}}$ & $177(19)^{\mathrm{a}}$ & $1234(360)^{\mathrm{a}}$ & $77(5)^{\mathrm{a}}$ & 1.4 \\
nanopaper & $9(0.4)^{\mathrm{c}}$ & $1.7(0.2)^{\mathrm{c}}$ & $111(7)^{\mathrm{c}}$ & $101(24)^{\mathrm{c}}$ & $104(3)^{\mathrm{c}}$ & 1.28 \\
\hline
\end{tabular}

230 *Means that are marked by different superscript letters within the same column are significantly different at 5\% level based on the one 231 way ANOVA.

\section{$232 \quad 3.4 \quad$ Morphology}

233 The reference was transparent but modified nanopaper was translucent (Figure 6); which might indicate 234 the presence of separate phases of lactic acid and nanocellulose (Yang et al. 1996). Another possible 235 reason can be that the modified nanopaper is porous and trapped air. The difference in density indicate 236 the slight porosity. The reference nanopaper had density of $1.34 \mathrm{gm} / \mathrm{cm}^{3}$ and modified nanopaper had $237 \quad 1.28 \mathrm{gm} / \mathrm{cm}^{3}$. 

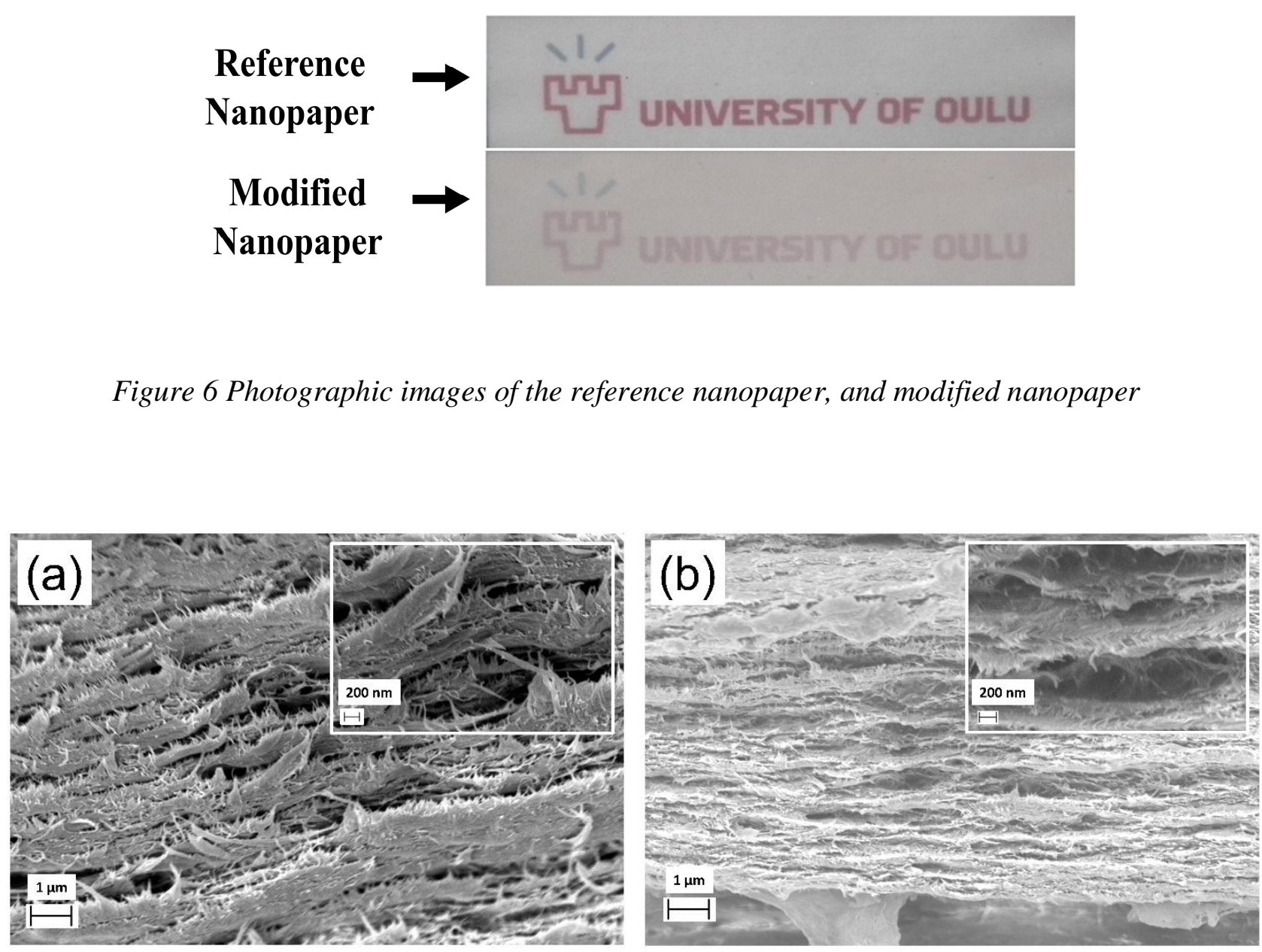

Figure 7 FESEM micrographs of fractured surface from tensile samples of (a)Reference nanopaper and (b)Modified nanopaper. Two different scales are shown: the bigger image represents the coarser scale $(1 \mu \mathrm{m})$ and inset image represents finer scale $(200 \mathrm{~nm})$. The reference material with typical layered structure of the nanopaper with the individual fibers. In contrast, modified nanopapers have the individual fibers and layers tightly glued to each other due to esterification.

247 Both reference and modified nanopaper had layered structure Figure 7 ( $a$ and $b$ ), which is due to

248 "concentration induced aggregation and floc formation" during filtration (Benítez et al. 2013). The

249 reference has loosely adhered layers, indicating the debonding between the layers under the tensile

250 load, which is likely due to breaking of inter layer hydrogen bonds. Additionally, the fracture

251 mechanism is debonding of layers along with pull out of fibers. Small fibrils can be seen protruding

252 from inset image of reference, no such fibrils are present in modified nanopaper. In modified 


\section{Page 15 of 21}

253 nanopapers, fibrils appears to be tightly glued which indicates lack of slipping. This slipping can be 254 attributed to high elongation of around 8\% (Figure 7), which is missing in modified nanopaper. The 255 modified nanopaper, on the other hand, has compact structure in which layers are tightly adhered to 256 each other (Figure 7 (b)). The esterification of surface, and LA moieties are binding the layers to each 257 other which is the reason for enhanced mechanical properties (Figure 4). Additionally, the fracture 258 mechanism seems to more brittle as no fibrils are bulging from the surface (compared to reference) and 259 nanofibers are in a way glued to each other. This might be the reason for brittle fracture, as they are 260 unable to slide and fracture from cross-section instead of pull out.

$261 \quad 3.5 \quad$ Effect of moisture and water content

262 The modified nanopaper was more resistant to moisture absorption from atmospheric humidity as 263 compared to nanopaper (Figure 8 (a)). It absorbed $43 \%$ less moisture compared to reference nanopaper, 264 indicating the hydrophobicity of samples. It has been mentioned that fewer hydroxyl group on the 265 surface of CNFs are accessible in the presence of polymer (oligomer) leading to lower moisture 266 absorption (Henriksson and Berglund 2007). Additionally, Figure 8 (b) presents the graph depicting 267 amount of water absorbed by samples when soaked in water. The trend is corresponding to moisture 268 content results; modified nanopaper are considerably hydrophobic than reference. The modified 269 nanopaper has $35 \%$ less water after 18 hours of absorption. 
(a)

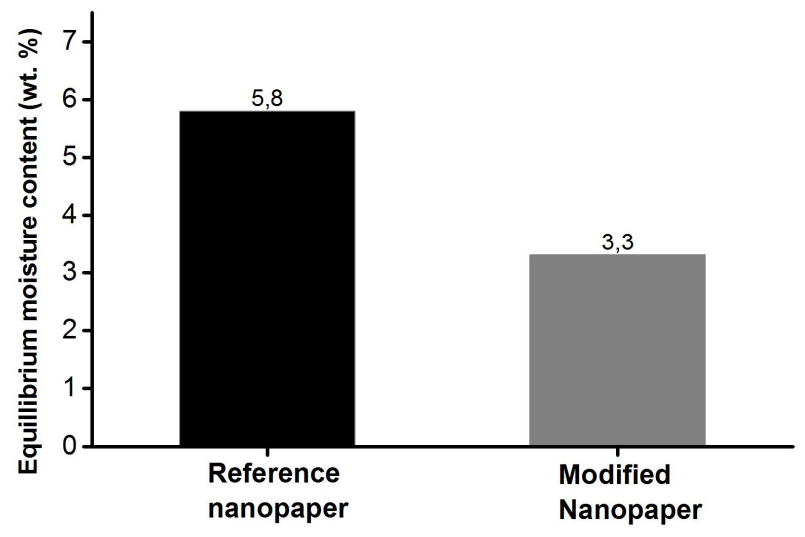

(b)

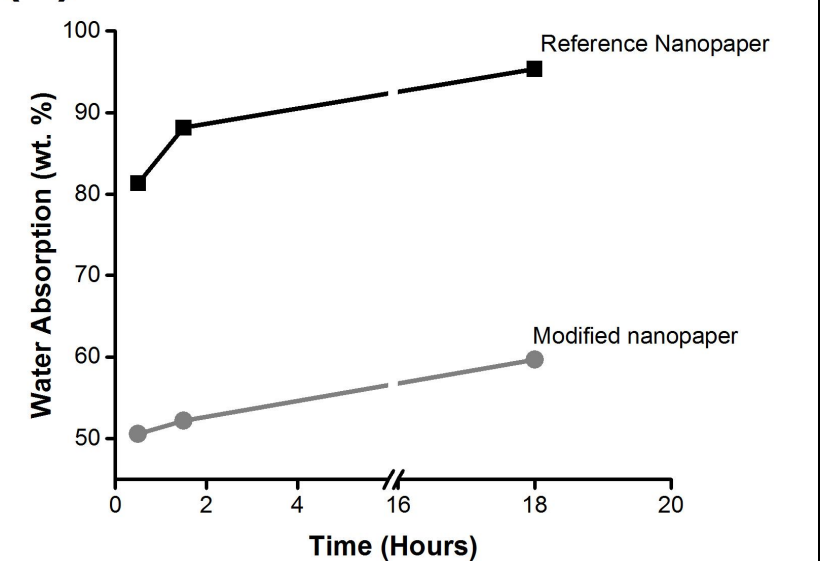

270 Figure 8 (a) Moisture content of reference nanopaper and modified nanopapers after storing at $20^{\circ} \mathrm{C}$ 271 temperature and RH 50 \% for 96 hours; reference has considerably high amount of moisture uptake 272 than the modified nanopaper indicating the hydrophobic nature and, (b) Amount of water absorption 273 as a function of time by reference nanopaper and modified nanopaper when soaked under water

274 The modified nanopaper has better mechanical performance under humidity (Figure 9 (a)), when 275 compared to reference nanopaper. It can be observed that humidity has devastating effect on stiffness 276 of nanocellulose paper, which has been reported in literature (Benítez et al. 2013); however, reference 277 nanopaper showed an interesting behavior that at relative humidity of around $75 \%$, it has a sharp drop 278 in storage modulus which indicates the sample loses its stiffness suddenly. A reason might be that the 279 water molecules are penetrating inside the material destroying the structure and acting as plasticizer, 280 resulting in loss in storage modulus (Sehaqui et al. 2014). The results are in agreement with (Benítez et 281 al. 2013), who also reported a steep drop in mechanical properties of nanopaper from $80 \%$ RH to $95 \%$

282 RH. The modified nanopaper has higher storage modulus than reference over the entire range of 283 humidity. 


\section{Page 17 of 21}
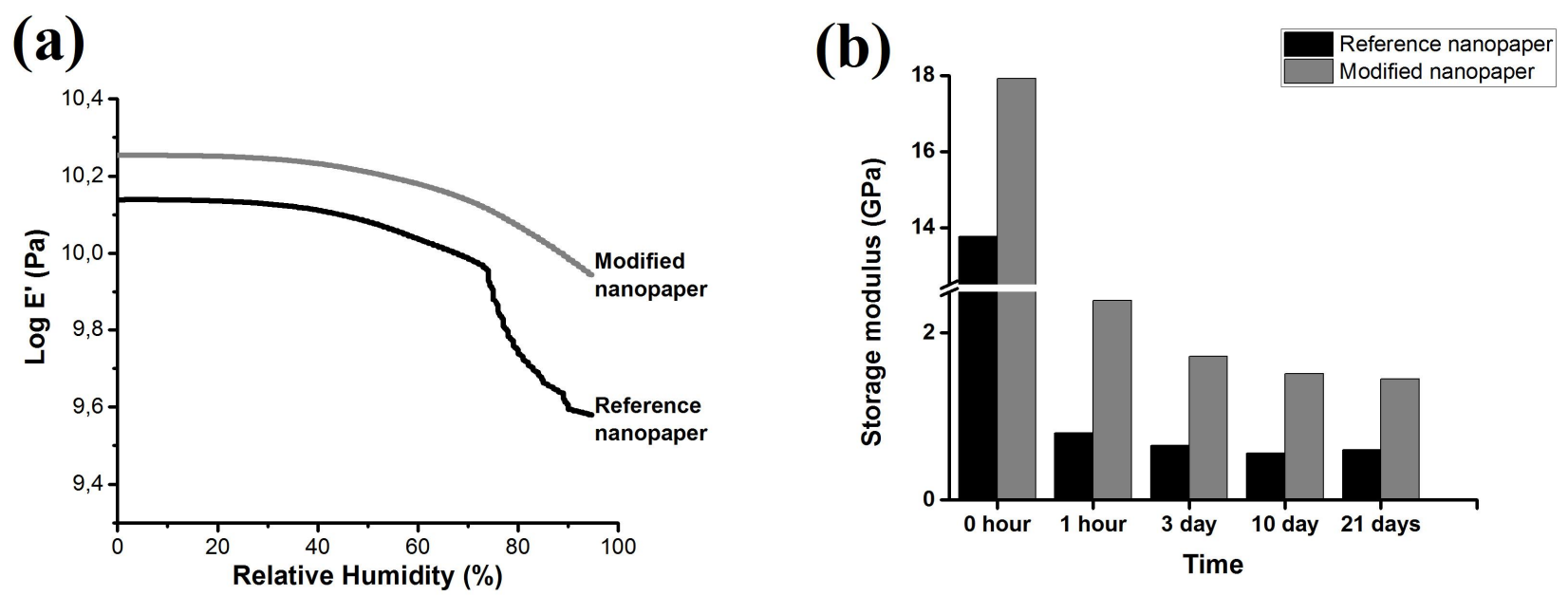

284 Figure 9 (a) Variation of storage modulus with respect to relative humidity of modified nanopaper and 285 reference nanopaper. (b) Evolution of wet storage modulus from of modified nanopaper and reference 286 nanopaper, when kept in water for extended period of time

287 Figure 9 (b) presents the evolution of wet storage modulus of water soaked modified nanopaper and 288 reference after various time intervals. It can be observed that there is a huge drop in modulus in both 289 reference and modified nanopaper; however, the modified nanopaper have superior properties in wet 290 state as the storage modulus is three times that of reference nanopaper even when samples are soaked 291 in water for 21 days. The water affects the mechanical properties in two ways, by plasticizing the 292 amorphous regions and by affecting hydrogen bonding among the nanofibrils (Benítez et al. 2013).

293 This gives an understanding about our results. In modified nanopapers, the humidity was able to 294 plasticize the amorphous region of cellulosic domains; however, due to presence of LA moieties at 295 interface, it did not alleviate the bonding between fibril as it does in reference. Hence, the modified 296 nanopaper was able to maintain higher stiffness when compared to reference. The results indicate that 297 modified nanopaper has far better performance than reference under the influence of water.

$298 \quad 3.6 \quad$ Thermal stability

299 Figure 10 shows that modified nanopapers are more thermally stable than the reference nanopaper. The 300 reference nanopaper lost $5 \mathrm{wt} \%$ of weight at $147^{\circ} \mathrm{C}$. On the other hand, modified nanopaper took 279 
$301{ }^{\circ} \mathrm{C}$, almost twice the temperature taken by reference (90\% higher) to lose $5 \%$ of weight. It is worth

302 mentioning that the results have been normalized after removing the amount of moisture in the

303 samples. Esterified nanocellulose has been reported to have better thermal stability than reference

304 (Agustin et al. 2016). It is worth noticing that at temperatures higher than $310^{\circ} \mathrm{C}$, reference has slower 305 degradation than modified samples which can be as a result from steeper degradation of LA phase.

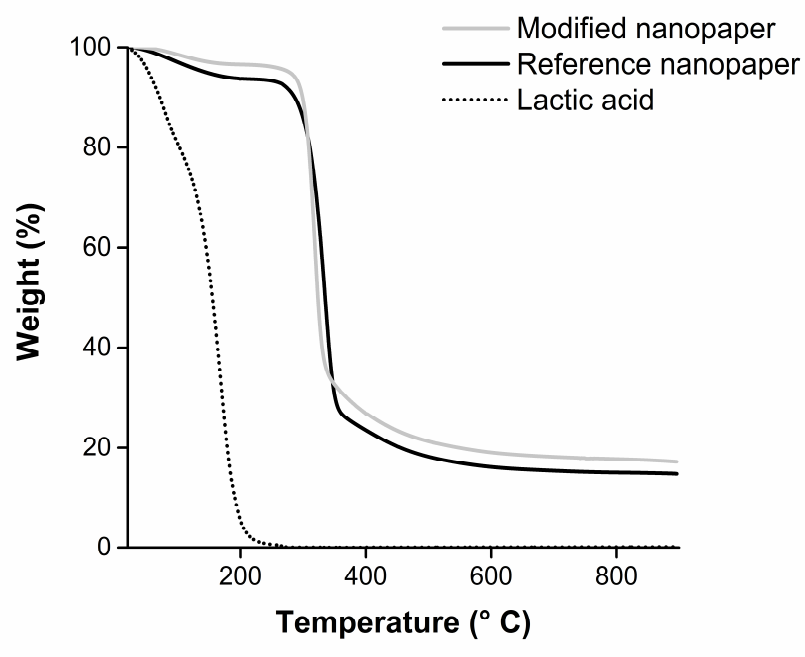

308 Figure 10 Thermogravimetry results indicating thermal stability of modified nanopaper along with 309 reference nanopaper and lactic acid; the results have been normalized after removing moisture 310 content. Modified nanopaper has higher stability than reference nanopape. Lactic acid thermogram is 311 also included.

3124 Conclusion

313 This study presents a novel approach to prepare modified nanopapers with enhanced properties. Lactic

314 acid monomer in aqueous medium was used, along with aid of ultrasonication and compression

315 molding. The modified nanopaper has higher modulus and yield strength, however, it lost the tensile

316 strength. Additionally, the modified nanopaper performed superiorly under humid environment and

317 presence of water. At $95 \%$ RH the storage modulus of modified nanopaper was three times that of 


\section{Page 19 of 21}

318 reference. Similar results were obtained for water soaked samples. Finally, the modified nanopaper was

319 thermally stable than when compared to reference nanopaper. The effect of parameters such as

320 sonication, temperature and catalyst is currently being pursued.

\section{Acknowledgements}

322 The authors acknowledge the financial support of the TEKES FiDiPro Program. Authors would also

323 like to thank Dr. Petteri Piltonen for his valuable feedback during the writing of this paper.

\section{References}

325 Agustin M B, Nakatsubo F, Yano H (2016) Products of low-temperature pyrolysis of nanocellulose esters and implications for the mechanism of thermal stabilization. Cellulose 23:2887-2903. doi: crystallinity in wood pulp. Pap Ja Puu 65:475-480.

330 Ballard CC, Broge EC, ller RK, et al (1961) Esterification of the Surface of Amorphous Silica. J Phys Chem 65:20-25. doi: 10.1021/j100819a007

Benítez AJ, Torres-Rendon J, Poutanen M, Walther A (2013) Humidity and multiscale structure govern mechanical properties and deformation modes in films of native cellulose nanofibrils. Biomacromolecules 14:4497-4506. doi: 10.1021/bm401451m

French AD (2014) Idealized powder diffraction patterns for cellulose polymorphs. Cellulose 21:885-

$$
\text { 896. doi: 10.1007/s10570-013-0030-4 }
$$

Habibi Y (2014) Key advances in the chemical modification of nanocelluloses. Chem Soc Rev 43:1519- 


\section{Page 20 of 21}

containing melamine formaldehyde. J Appl Polym Sci 106:2817-2824. doi: 10.1002/app.26946

341 Kobayashi S, Uyama H, Suda S, Namekawa S (1997) Dehydration polymerization in aqueous medium $342 \quad$ catalyzed by lipase. Chem Lett 26:105.

343 Lee K-Y, Aitomäki Y, Berglund LA, et al (2014) On the use of nanocellulose as reinforcement in polymer 344 matrix composites. Compos Sci Technol 105:15-27. doi: 10.1016/j.compscitech.2014.08.032 345 Lönnberg H, Zhou Q, III HB, et al (2006) Grafting of Cellulose Fibers with Poly ( E -caprolactone ) and 346 Poly ( L -lactic acid ) via Ring-Opening Polymerization. Biomacromolecules 7:2178-2185.

Peltzer M , Pei A, Zhou Q, et al (2014) Surface modification of cellulose nanocrystals by grafting with poly(lactic acid). Polym Int 63:1056-1062. doi: 10.1002/pi.4610

Przybysz P, Dubowik M, Kucner MA, et al (2016) Contribution of Hydrogen Bonds to Paper Strength Properties. PLoS One 11:e0155809.

Sehaqui H, Ezekiel M ushi N, M orimune S, et al (2012) Cellulose nanofiber orientation in nanopaper and nanocomposites by cold drawing. ACS Appl Mater Interfaces 4:1043-1049. doi: $10.1021 / \operatorname{am} 2016766$

Sehaqui H, Zimmermann T, Tingaut P (2014) Hydrophobic cellulose nanopaper through a mild esterification procedure. Cellulose 21:367-382. doi: 10.1007/s10570-013-0110-5

Suslick KS (2000) Sonochemistry. In: Kirk-Othmer Encyclopedia of Chemical Technology. John Wiley \& Sons, Inc.,

Tanaka H, Kurihashi T (2003) Synthesis of Polyesters by Emulsion Polycondensation Reaction in Water. 


\section{Page $\mathbf{2 1}$ of $\mathbf{2 1}$}

Polym J 35:359-363. doi: 10.1295/polymj.35.359

363 Teramoto Y, Yoshioka M, Shiraishi N, Nishio Y (2002) Plasticization of cellulose diacetate by graft

364 copolymerization of $\varepsilon$-caprolactone and lactic acid. J Appl Polym Sci 84:2621-2628. doi:

$365 \quad 10.1002 /$ app.10430

366 Tingaut P, Zimmermann T, Sèbe G (2012) Cellulose nanocrystals and microfibrillated cellulose as

367 building blocks for the design of hierarchical functional materials. J Mater Chem 22:20105. doi:

$368 \quad 10.1039 /$ c2jm32956e

369 Tischer PCSF, Sierakowski MR, Westfahl H, Tischer CA (2010) Nanostructural reorganization of

370 bacterial cellulose by ultrasonic treatment. Biomacromolecules 11:1217-1224. doi:

$371 \quad 10.1021 /$ bm901383a

372 Tjeerdsma BF, M ilitz H (2005) Chemical changes in hydrothermal treated wood: FTIR analysis of 373 combined hydrothermal and dry heat-treated wood. Holz als Roh - und Werkst 63:102-111. doi: $374 \quad 10.1007 / \mathrm{s} 00107-004-0532-8$

375 Wang S, Cheng Q (2009) A novel process to isolate fibrils from cellulose fibers by high-intensity $376 \quad$ ultrasonication, Part 1: Process optimization. J Appl Polym Sci 113:1270-1275. doi:

$377 \quad 10.1002 / a p p .30072$

378 YangJ, Winnik M A, Ylitalo D, Devoe RJ (1996) Polyurethane-Polyacrylate Interpenetrating Networks.

379 1. Preparation and Morphology. Macromolecules 9297:7047-7054. doi: 10.1021/ma9601373

380 Yoo DK, Kim D, Lee DS (2006) Synthesis of lactide from oligomeric PLA: Effects of temperature, 381 pressure, and catalyst. Macromol Res 14:510-516. doi: 10.1007/BF03218717 